\title{
OH masers towards IRAS 19092+0841
}

\author{
K. A. Edris ${ }^{1,2}$, G. A. Fuller ${ }^{1}$, S. Etoka ${ }^{3}$, and R. J. Cohen ${ }^{1}$ \\ 1 Jodrell Bank Centre for Astrophysics, School of Physics and Astronomy, Alan Turing Building University of Manchester, \\ Manchester M13 9PL, UK \\ 2 Al-Azhar University, Faculty of Science, Astronomy Department, PO Box 11884 Naser City, Cairo, Egypt \\ e-mail: khedres@azhar.edu.eg \\ ${ }^{3}$ Hamburger Sternwarte, Gojenbergsweg 112, 21029 Hamburg, Germany
}

Received 21 July 2016 / Accepted 26 July 2017

\begin{abstract}
Context. Maser emission is a strong tool for studying high-mass star-forming regions and their evolutionary stages. OH masers in particular can trace the circumstellar material around protostars and determine their magnetic field strengths at milliarcsecond resolution.

Aims. We seek to image $\mathrm{OH}$ maser emission towards high-mass protostellar objects to determine their evolutionary stages and to locate the detected maser emission in the process of high-mass star formation.

Methods. In 2007, we surveyed OH maser emission towards 217 high-mass protostellar objects to study its presence. In this paper, we present follow-up MERLIN observations of a ground-state OH maser emission towards one of these objects, IRAS $19092+0841$. Results. We detect emissions from the two $\mathrm{OH}$ main spectral lines, 1665 and $1667 \mathrm{MHz}$, close to the central object. We determine the positions and velocities of the $\mathrm{OH}$ maser features. The masers are distributed over a region of $\sim 5^{\prime \prime}$ corresponding to $22400 \mathrm{AU}$ (or $\sim 0.1 \mathrm{pc}$ ) at a distance of $4.48 \mathrm{kpc}$. The polarization properties of the $\mathrm{OH}$ maser features are determined as well. We identify three Zeeman pairs from which we inferred a magnetic field strength of $\sim 4.4 \mathrm{mG}$ pointing towards the observer.

Conclusions. The relatively small velocity spread and relatively wide spacial distribution of the $\mathrm{OH}$ maser features support the suggestion that this object could be in an early evolutionary state before the presence of disk, jets or outflows.
\end{abstract}

Key words. masers - molecular data - stars: formation - stars: protostars - ISM: individual objects: IRAS 19092+0841 polarization

\section{Introduction}

Observations show that $\mathrm{OH}$ masers are associated with different evolutionary stages of star-forming regions. It was believed that they are only associated with HII regions (e.g. Garay \& Lizano 1999). However, observations that have been carried out towards a number of star-forming regions show that $\mathrm{OH}$ masers are also associated with an earlier stage before the appearance of ionized HII regions (e.g. Cohen et al. 1988; Braz et al. 1990). This later type of $\mathrm{OH}$ maser is associated with an accretion phase, outflow, and circumstellar disks (Brebner et al. 1987; Hutawarakorn \& Cohen 1999; Hutawarakorn et al. 2002; Fuller et al. 2001; Edris et al. 2005). An OH maser survey by Edris et al. (2007, hereafter EFC07) detected the $\mathrm{OH}$ masers towards $26 \%$ of a sample of 217 high-mass protostellar objects (HMPOs) candidates. It is of interest to know the distributions of the $\mathrm{OH}$ masers and their associations towards these regions in addition to their position in the evolutionary sequence. Maser emission gives a unique opportunity of observing those typically far regions in some detail. This paper is the second follow up of the EFC07 survey with a high angular resolution observation. One of EFC07 objects (IRAS 20126+4104; hereafter IRAS 20126) was studied by Edris et al. (2005) in the ground-state $\mathrm{OH}, 22-\mathrm{GHz} \mathrm{H}_{2} \mathrm{O}$, and 6.7- $\mathrm{GHz}$ class II $\mathrm{CH}_{3} \mathrm{OH}$ masers lines.

The present object of study, IRAS 19092+0841 (hereafter IRAS 19092), is one of the HMPOs sample studied by Palla et al. (1991) and Molinari et al. (1996, 1998, 2000). Palla et al. (1991) divided this sample of 260 IRAS sources into two subsamples of so-called high and low sources. The high sources are the sources located in the "higher" part of the colour-colour diagram such that $[25-12]>=0.57$, which in essence is the prescription for the presence of associated ultra-compact HII (UCHII) regions according to Wood \& Churchwell (1989) criteria. On the other hand, the low sources are most probably made of two groups of sources with a distinct evolutionary state (Molinari et al. 1996). The first low group of sources is believed to be at a very early evolutionary state before the creation of a real young stellar object at the centre and consequently before the appearance of any detectable UCHII regions. The other low group of sources is believed to contain more evolved sources (older than the high sources) containing objects that have already dispersed much of their circumstellar material. IRAS 19092 belongs to the latter group of the low subsample. IRAS 19092 is at an estimated distance of $4.48 \mathrm{kpc}$ (Molinari et al. 1996) and has a luminosity of $10^{4} L_{\odot}$. An ammonia core was found towards IRAS 19092 at velocity $\sim 58 \mathrm{~km} \mathrm{~s}^{-1}$ (Molinari et al. 1996).

IRAS 19092 is associated with different types of maser emission. $\mathrm{OH}$ maser was originally reported by MacLeod et al. (1998) and then by EFC07. Water maser was detected by Palla et al. (1991). IRAS 19092 is also associated with the class I methanol masers emission at $44 \mathrm{GHz}$, but it is not associated with maser emission at $95 \mathrm{GHz}$ (Kurtz et al. 2004; Fontani et al. 2010). Class II $\mathrm{CH}_{3} \mathrm{OH}$ masers at $6.7 \mathrm{GHz}$ were detected by Szymczak et al. (2000) and mapped by Pandian et al. (2011). CO and $\mathrm{H}_{2}$ observations failed to detect any sign of outflows in this region (Zhang et al. 2005; Varricatt et al. 2010). IRAS 19092 
Table 1. Observing and calibration parameters for the MERLIN spectral-line observations of IRAS 19092.

\begin{tabular}{cc}
\hline \hline Observational parameters & OH masers \\
\hline Date of observation & 4 and 5 April 2003 \\
Antenna used & seven antenna \\
Field centre (2000) & $\alpha=19^{\mathrm{h}} 11^{\mathrm{m}} 37.40^{\mathrm{s}}$ \\
& $\delta=08^{\circ} 46^{\prime} 30.00^{\prime \prime}$ \\
Rest frequency (MHz) & 1665.402 \\
& 1667.359 \\
No. of frequency channels & 512 \\
Total bandwidth (MHz) & 0.5 \\
bandpass calibrator & $3 \mathrm{C} 84$ \\
Polarization angle calibrator & $3 \mathrm{C} 286$ \\
Phase calibrator & $1919+086$ \\
\hline
\end{tabular}

is not associated with a close $\mathrm{cm}$ radio continuum emission. The closest $\mathrm{cm}$ continuum emission detected by Molinari et al. (1998) at $6 \mathrm{~cm}$ is offset from the IRAS position by 110 arcsec. However, IRAS 19092 is associated with mm continuum emission detected by Molinari et al. (2000) and the Bolocam Galactic Plane Survey (BGPS; Rosolowsky et al. 2010).

To determine what the $\mathrm{OH}$ masers trace and how they are distributed and related to other tracers, IRAS 19092 has been observed at high angular resolution using MERLIN. The details of the observations and reduction are given in Sect. 2 and the results are presented in Sect. 3. In Sect. 4 we discuss the interpretation while conclusions are drawn in Sect. 5.

\section{Observations and data reduction}

Table 1 gives the parameters for the MERLIN observations. The phase calibrator source $1919+086$ was used to retrieve the absolute position of the maser components and therefore compare their locations from one line to another. A bandpass calibrator 3C 84 was observed to calibrate the variation of instrumental gain and phase across the spectral bandpass. Observations of 3C 286 were also made during the observing run, with the same correlator configuration and bandwidth, to calibrate for the polarization angle.

IRAS 19092 was observed in the 1665- and 1667-MHz OH maser transitions in April 2003 using the seven telescopes of the MERLIN network available at that time. During the observations, the frequency was cycled between the two $\mathrm{OH}$ line frequencies to provide data on both transitions spread over the whole observing track. The velocity resolution was $0.21 \mathrm{~km} \mathrm{~s}^{-1}$ for a total of $0.25 \mathrm{MHz}$ spectrum bandwidth corresponding to a $45 \mathrm{~km} \mathrm{~s}^{-1}$ velocity range. The observations were performed in full polarization mode. The removal of obvious bad data and correction for gain-elevation effects were performed using the MERLIN d-programs (see Diamond et al. 2003). The flux density of the amplitude calibrator 3C 84 was determined by comparing the visibility amplitudes on the shortest baselines with those of 3C 286. Using flux density of $13.625 \mathrm{Jy}$ for 3C 286 (Baars et al. 1977), the flux density of 3C 84 at the time of the observation was determined to be $21 \mathrm{Jy}$.

In AIPS more refined editing was performed and the data were calibrated for all remaining instrumental and atmospheric effects. Starting from a point source model, the phase calibrator was mapped with a total of three rounds of phase self-calibration and the resulting corrections were applied to the source data. The polarization leakage for each antenna was determined using 3C 84 and the polarization position angle correction was performed using 3C 286. Maps of the LHC and RHC emission were produced, and finally the maps of the Stokes parameters $(I, Q, U$, and $V$ ) were produced using clean algorithms in AIPS. The rms noise, after CLEANing, was typically $14 \mathrm{mJy} /$ beam and the FWHM of the restoring beam is $0.9 \times 0.4$ arcsec at a position angle of $-31^{\circ}$.

The individual channel maps usually showed one, two, or three unresolved components, and each component was usually seen across several spectral channels. The positions of the maser components were determined by fitting two-dimensional Gaussian components to the brightest peaks in each channel map. Components were considered as spectral features if they occurred in three or more consecutive channels and were then grouped into spectral features. The positions and velocities of these maser features were obtained by taking flux weighted means over those channels showing emission from the feature. The uncertainties in relative positions are given in Table 2, while the absolute positional accuracy is estimated as described in Edris et al. (2005) to be better than 30 mas. This positional uncertainty depends on four factors: the position accuracy of the phase calibrator, accuracy of the telescope positions, relative position error depending on the beam size and signal-to-noise ratio and, finally, atmospheric variability.

\section{Results}

The 1665- and $1667-\mathrm{MHz} \mathrm{OH}$ maser lines were detected with MERLIN. The absolute position of the brightest maser feature in the $1665 \mathrm{MHz}$ OH line is $19^{\mathrm{h}} 11^{\mathrm{m}} 38.974^{\mathrm{s}}+08^{\circ} 46^{\prime} 31.09^{\prime \prime}$ at velocity $58 \mathrm{~km} \mathrm{~s}^{-1}$. Radial velocities, here and elsewhere, are given relative to the local standard of rest (LSR).

A total of 11 ( 9 in the $1665-\mathrm{MHz}$ line and 2 in the $1667-\mathrm{MHz}$ line) $\mathrm{OH}$ maser features were detected. At the $1665-\mathrm{MHz}$ line, there are 5 left-hand circular polarization (LHC) and 4 righthand circular polarization (RHC) features while the two features detected at the $1667-\mathrm{MHz}$ line are LHC. Table 2 presents the parameters of the $\mathrm{OH}$ maser features detected, namely the velocities, peak intensities, and positions for each hand of circular polarization. The label $\mathrm{Z}$ identifies the left-hand and righthand polarized features of a Zeeman pair. These Zeeman pairs were identified by searching Tables 2 and 3 for groups of features of opposite polarization that coincide to within the positional uncertainties. Three possible Zeeman pairs were identified, which indicates that the $1665 \mathrm{MHz}$ OH line in IRAS 19092 experience zeeman splitting of several $\mathrm{km} \mathrm{s}^{-1}$ exceeding the line width (less than $1 \mathrm{~km} \mathrm{~s}^{-1}$ ). The strength of the magnetic field can be measured from the velocity difference between the two hands of polarization (Elitzur 1996). The splitting of +2.49 to $+2.67 \mathrm{~km} \mathrm{~s}^{-1}$ between the left- and right-hand polarized features of the 3 Zeeman pairs Z1-Z3 (cf. Table 2) leads to a magnetic field strengths ranging from 4.2 to $4.6 \mathrm{mG}$ pointing towards us. The magnetic field measured towards IRAS 19092 is very similar to what was measured towards other star-forming regions such as W3(OH) (Garcia-Barreto et al. 1988), W75N (Hutawarakorn et al. 2002) and W51 (Etoka et al. 2012) harboring massive central objects at early stages of the star-forming process.

Figure 1 shows the map of the $\mathrm{OH}$ maser compared to the $1.1 \mathrm{~mm}$ map from Faustini et al. (2009). The $\mathrm{OH}$ maser are spread over a region of $\sim 5^{\prime \prime}$ (or 17" if the far northern $1665 \mathrm{MHz}$ feature 5 is included) corresponding to $22400 \mathrm{AU}$ (or $\sim 0.1 \mathrm{pc}$ ) at a distance of $4.48 \mathrm{kpc}$. The two features of the $1667-\mathrm{MHz}$ line (Table 2$)$ are closely associated ( $\leq 300$ mas) with the lowest declination components at $1667 \mathrm{MHz}$ of relatively weak intensity. 
K. A. Edris et al.: OH masers towards IRAS 19092+0841

Table 2. Parameters of the left- and right-hand circular polarization features of $1665-\mathrm{MHz}$ and the $1667-\mathrm{MHz} \mathrm{OH}$ masers detected towards IRAS 19092.

\begin{tabular}{|c|c|c|c|c|c|c|c|c|}
\hline \multirow[t]{2}{*}{ No. } & \multirow{2}{*}{$\begin{array}{c}\text { Vel. } \\
\mathrm{km} \mathrm{s}^{-1}\end{array}$} & \multirow{2}{*}{$\begin{array}{c}\text { Peak intensity } \\
\text { Jy beam }^{-1}\end{array}$} & RA & \multirow{2}{*}{$\begin{array}{c}\text { Error } \\
\mathrm{S} \\
\end{array}$} & \multirow{2}{*}{$\begin{array}{c}\text { Dec } \\
0, \prime \prime\end{array}$} & \multirow{2}{*}{$\begin{array}{c}\text { Error } \\
\prime \prime\end{array}$} & \multirow{2}{*}{$\begin{array}{c}\text { Zeeman } \\
\text { pair }\end{array}$} & \multirow{2}{*}{$\begin{array}{c}B \\
\mathrm{mG} \\
\end{array}$} \\
\hline & & & $\mathrm{h} \mathrm{m} \mathrm{s}$ & & & & & \\
\hline \multicolumn{9}{|c|}{$1665-\mathrm{MHz}$} \\
\hline \multicolumn{9}{|c|}{ LHC } \\
\hline 1 & 60.65 & $0.17 \pm 0.01$ & 191139.002 & 0.004 & 84630.38 & 0.12 & $\mathrm{Z} 1$ & -4.6 \\
\hline 2 & 60.59 & $0.77 \pm 0.01$ & 191138.973 & 0.000 & 84631.10 & 0.01 & $\mathrm{Z2}$ & -4.3 \\
\hline 3 & 60.70 & $0.05 \pm 0.01$ & 191138.899 & 0.008 & 84628.33 & 0.18 & & \\
\hline 4 & 60.58 & $0.18 \pm 0.01$ & 191138.943 & 0.001 & 84627.68 & 0.03 & $\mathrm{Z3}$ & -4.2 \\
\hline 5 & 60.50 & $0.10 \pm 0.01$ & 191138.909 & 0.003 & 84644.80 & 0.06 & & \\
\hline \multicolumn{9}{|l|}{ RHC } \\
\hline 6 & 57.98 & $0.48 \pm 0.02$ & 191139.001 & 0.003 & 84630.44 & 0.07 & $\mathrm{Z} 1$ & \\
\hline 7 & 58.08 & $1.53 \pm 0.02$ & 191138.974 & 0.000 & 84631.09 & 0.00 & $\mathrm{Z} 2$ & \\
\hline 8 & 58.07 & $0.14 \pm 0.02$ & 191138.941 & 0.006 & 84627.95 & 0.15 & & \\
\hline 9 & 58.09 & $0.25 \pm 0.02$ & 191138.950 & 0.001 & 84627.59 & 0.02 & $\mathrm{Z3}$ & \\
\hline \multicolumn{9}{|c|}{ 1667-MHz } \\
\hline \multicolumn{9}{|c|}{ LHC } \\
\hline 1 & 60.67 & $0.48 \pm 0.01$ & 191138.950 & 0.001 & 84627.81 & 0.03 & & \\
\hline 2 & 60.55 & $0.40 \pm 0.01$ & 191138.944 & 0.002 & 84627.66 & 0.04 & & \\
\hline
\end{tabular}

Notes. The values in Cols. 5 and 7 refer to the relative position error while the absolute position uncertainty is $\sim 30$ mas (cf. Sect. 2).

Table 3. Stokes and polarization parameters of the 1665- and 1667-MHz OH masers features detected towards IRAS 19092.

\begin{tabular}{ccccccccccc}
\hline \hline No. & $\begin{array}{c}\text { Vel. } \\
\mathrm{km} \mathrm{s}^{-1}\end{array}$ & $\begin{array}{c}I^{a} \\
\mathrm{Jy} \mathrm{beam}^{-1}\end{array}$ & $\begin{array}{c}Q^{b} \\
\mathrm{Jy} \mathrm{beam}^{-1}\end{array}$ & $\begin{array}{c}U^{c} \\
\mathrm{Jy} \mathrm{beam}^{-1}\end{array}$ & $\begin{array}{c}V^{d} \\
\mathrm{Jy} \mathrm{beam}^{-1}\end{array}$ & $\begin{array}{c}P^{e} \\
\mathrm{Jy} \mathrm{beam}^{-1}\end{array}$ & $\begin{array}{c}\chi^{f} \\
\circ\end{array}$ & $\begin{array}{c}m_{\mathrm{L}}^{g} \\
\%\end{array}$ & $\begin{array}{c}m_{\mathrm{C}}^{h} \\
\%\end{array}$ & $\begin{array}{c}m_{\mathrm{T}}^{k} \\
\%\end{array}$ \\
\hline $\begin{array}{c}1665-\mathrm{MHz} \\
1\end{array}$ & 60.65 & 0.05 & 0.00 & 0.02 & -0.04 & 0.02 & - & 33.9 & -81.4 & 88.2 \\
2 & 60.59 & 0.44 & 0.04 & 0.07 & -0.32 & 0.08 & 30.1 & 18.1 & -71.2 & 73.5 \\
3 & 60.70 & 0.04 & 0.00 & 0.02 & 0.00 & 0.02 & - & 49.2 & 3.8 & 49.3 \\
4 & 60.58 & 0.11 & 0.00 & 0.00 & -0.05 & 0.00 & - & 0.0 & -45.3 & 45.3 \\
5 & 60.50 & 0.05 & 0.00 & 0.00 & 0.00 & 0.00 & - & 0.0 & 0.0 & 0.0 \\
6 & 57.98 & 0.12 & 0.00 & -0.08 & 0.09 & 0.08 & - & 65.6 & 74.0 & 98.9 \\
7 & 58.08 & 1.23 & -0.12 & -0.71 & 0.47 & 0.72 & -40.2 & 58.3 & 38.5 & 69.8 \\
8 & 58.07 & 0.07 & 0.00 & -0.05 & 0.05 & 0.05 & - & 69.9 & 61.7 & 93.2 \\
9 & 58.09 & 0.22 & -0.03 & -0.15 & 0.07 & 0.16 & 39.4 & 70.1 & 31.3 & 76.8 \\
$1667-\mathrm{MHz}$ & & & & & & & & & & \\
1 & 60.67 & 0.33 & 0.02 & 0.02 & 0.02 & 0.03 & 22.5 & 9.1 & 6.1 & 10.9 \\
2 & 60.55 & 0.28 & 0.02 & 0.02 & 0.02 & 0.03 & 22.5 & 10.7 & 7.1 & 12.8 \\
\hline
\end{tabular}

Notes. ${ }^{(a)} I(u, v)=1 / 2[R R(u, v)+L L(u, v)],{ }^{(b)} Q(u, v)=1 / 2[R L(u, v)+L R(u, v)],{ }^{(c)} U(u, v)=1 / 2[L R(u, v)-R L(u, v)],{ }^{(d)} V(u, v)=1 / 2[R R(u, v)-$ $L L(u, v)],{ }^{(e)} P P O L=\left(\mathrm{Q}^{2}+\mathrm{U}^{2}\right)^{(1 / 2)},{ }^{(f)} P A N G=(0.5 \times \arctan (U / Q)) \times(180 / \pi),{ }^{(g)} m_{L}(\%)=100 \times P / I,{ }^{(h)} m_{C}(\%)=100 \times V / I,{ }^{(k)} m_{T}(\%)=$ $100 \times\left(Q^{2}+U^{2}+V^{2}\right)^{(1 / 2)} / I$.

Table 3 presents the Stokes parameters $I, Q, U$, and $V$, the polarization position angle $\chi$ (angles are measured from north towards east), the linearly polarized flux $P$, the percentage of linear polarization $m_{\mathrm{L}}$, the percentage of circular polarization $m_{\mathrm{C}}$, and the total percentage of polarization $m_{\mathrm{T}}$ of each feature. The Stokes intensities are shown as zero in this table if their flux is below the noise level. There are only two features that are not polarized.

\section{Discussion}

The observations reported towards the IRAS 19092 region suggest that it is a star-forming region in an early evolutionary state before the formation of HII region. It is not associated with neither $\mathrm{cm}$ continuum emission nor outflow signature (Molinari et al. 1998; Zhang et al. 2005; Varricatt et al. 2010). The velocity spread and distribution of the $\mathrm{OH}$ maser emission of the observations presented here may indicate that collapsing processes are dominant. The velocity extent is less than $3 \mathrm{~km} \mathrm{~s}^{-1}$ for an overall maser emission extent of $22400 \mathrm{AU}$. Wu et al. (2007) reported a close $\sim 10^{\prime \prime}$ south-west source with some spectral lines showing a blue profile expected to be the signature of an inflow motion. The weak flux density of the detected OH masers suggests that IRAS 19092 is in an early evolutionary state. The survey of EFC07 for $\mathrm{OH}$ masers towards high-mass protostellar objects found that most of the detected 


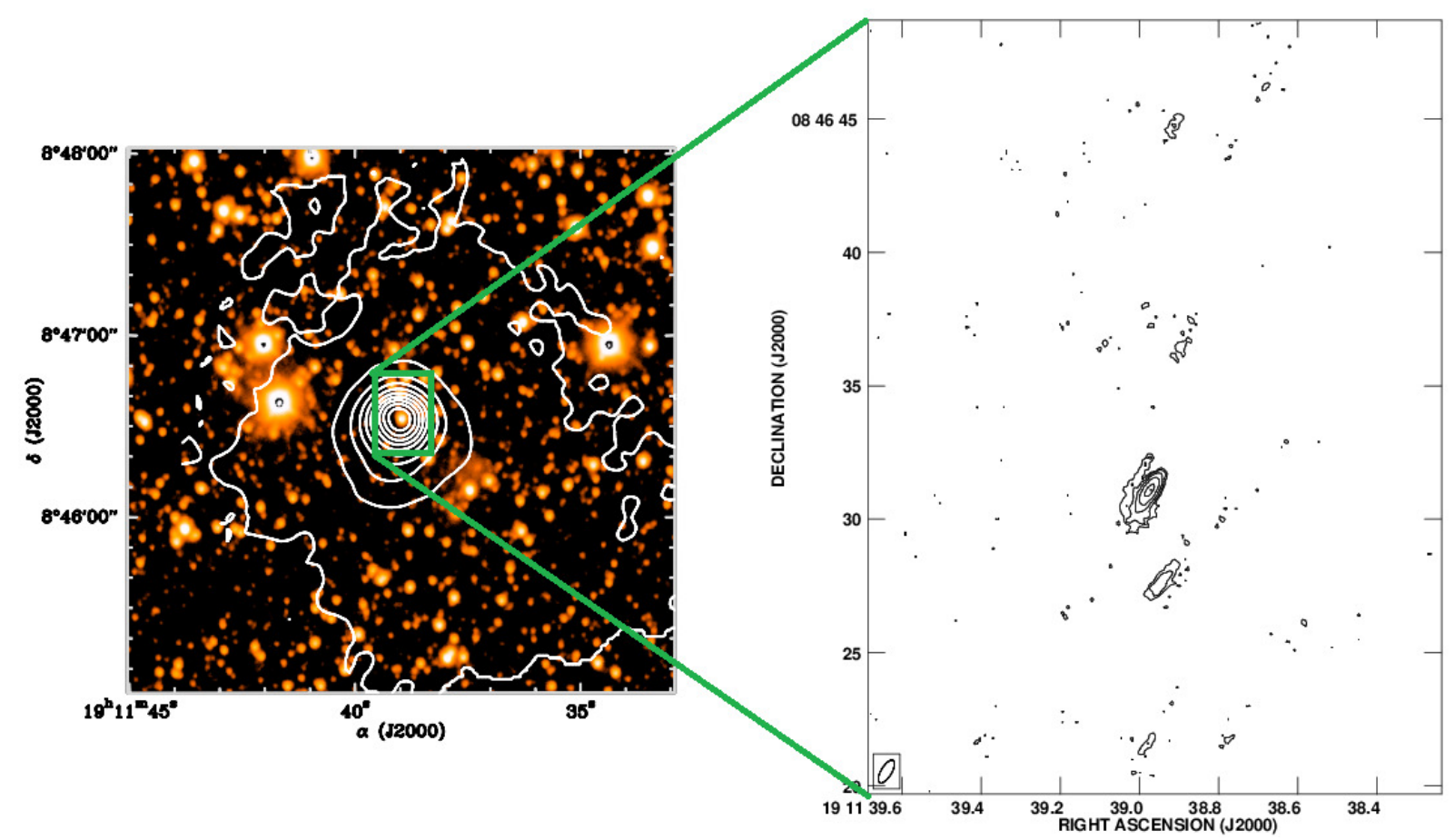

Fig. 1. Left panel: image for the IRAS 19092 region at the $K$ s band at $2.17 \mu \mathrm{m}$ with superimposed SCUBA $850 \mu \mathrm{m}$ continuum in white contours (from Faustini et al. 2009; Fig. A.16). The small box in the middle represent the area of 1665-MHz OH maser maps shown in the right panel. The $\mathrm{OH}$ masers peak flux is $7.24 \mathrm{Jy}_{\text {beam }^{-1}}$ and the contour levels are $0.12 \times(4,8,16,32,60,120)$.

sources show $\mathrm{OH}$ flux densities $<4.5 \mathrm{Jy}$, while the $\mathrm{OH}$ masers associated with HII regions show much higher flux densities (e.g. Gaume, \& Mutel 1987; Gasiprong et al. 2002). Fontani et al. (2006) reported the lack of deuterium towards this region and concluded that IRAS $19092+0841$ is associated with a cold and dense gas with chemical and physical conditions identical to those associated with low-mass starless cores. The presence of class I methanol masers (Kurtz et al. 2004) supports the suggestion of early evolutionary state. Ellingsen (2006) suggested that the class I methanol masers may signify an earlier stage of highmass star formation than the class II masers. The early evolutionary stage interpretation is also consistent with the suggestion of Molinari et al. (1996) that some of the sources in the low sample (to which IRAS 19092 is related; see Sect. 1) are in an earlier evolutionary state than their counterparts in the high sample.

\subsection{Comparison with other tracers}

The $\mathrm{OH}$ masers were previously detected by Macleod et al. (1998) and EFC07. There is no remarkable change between the three observations in the spectrum, velocity range, or peak velocity, but the flux density in the later survey of EFC07 was relatively stronger (3.45 Jy). Also in the survey of EFC07 the $1665 \mathrm{MHz}$ (at LHC) spectrum showed a weak feature at velocity $55 \mathrm{~km} \mathrm{~s}^{-1}$ that did not appear in the spectrum of Macleod et al. (1998) or in the present observations. This feature at $55 \mathrm{~km} \mathrm{~s}^{-1}$ has the same velocity as the class II methanol maser at $6.7 \mathrm{GHz}$ detected by Szymczak et al. (2000) and more recently by Fontani et al. (2010). In the $6.7 \mathrm{GHz}$ spectrum of Fontani et al. (2010) and Pandian et al. (2011), a new weaker methanol maser feature appears at velocity $\simeq 63 \mathrm{~km} \mathrm{~s}^{-1}$ (their Figs. A.2 and 1).

The velocities of the $\mathrm{OH}$ maser features are more consistent with those of the $44 \mathrm{GHz}$ class I than those of the $6.7 \mathrm{GHz}$ class II methanol masers. On the other hand, the $6.7 \mathrm{GHz}$ class II methanol maser component (centred at $V \sim 55 \mathrm{~km} \mathrm{~s}^{-1}$ ) do not coincide with any $\mathrm{OH}$ maser spectral features. The close association of $\mathrm{OH}$ (in particular the $1665 \mathrm{MHz}$ line) and class II methanol masers have been proposed by Caswell (1996) from subarcsec accuracy survey and modelled by Cragg et al. (2002). In some cases a disk has been suggested to be the source of the two maser types (Edris et al. 2005; Gray et al. 2003). However, a close association between $\mathrm{OH}$ masers and class I methanol masers towards sources in such early evolutionary state has never been reported so far. The flux ratio of these masers, $S(6668) / S(1665)=6 / 1.5=4$ and $S(44) / S(1.6)=1 / 1.5=0.6$, places IRAS 19092 in $\mathrm{OH}$-favoured sources. Although the $\mathrm{OH}$ and class $\mathrm{I} \mathrm{CH}_{3} \mathrm{OH}$ masers are associated, there is a clear difference in position, amounting to $2.7 \operatorname{arcsec}(\sim 0.06 \mathrm{pc})$, suggesting that the $\mathrm{OH}$ and class $\mathrm{I} \mathrm{CH}_{3} \mathrm{OH}$ masers are not co-propagating. The water masers associated with IRAS 19092 (Palla et al. 1991; Brand et al. 1994) peak at velocity $\simeq 57 \mathrm{~km} \mathrm{~s}^{-1}$ with a velocity range of $2.5 \mathrm{~km} \mathrm{~s}^{-1}$. This may indicate a more compact region than that of the $\mathrm{OH}$ masers.

The velocity of the strongest $\mathrm{OH}$ maser feature agrees with the gas velocity of the ammonia core measured by Molinari et al. (1996) and the peak velocity of the $C^{34} S$ observations (which is assumed to represent the velocity of high-density gas) carried out by Brand et al. (2001). This indicates that the $\mathrm{OH}$ masers emission originated from the core of this region, which is also consistent with the submm map of Faustini et al. (2009; Fig. 1).

The $\mathrm{OH}$ masers seem to arise from the core of the $850 \mu \mathrm{m}$ continuum emission. However, the relatively small velocity spread of the $\mathrm{OH}$ maser features despite their relatively large distributions of $5^{\prime \prime}$ (or 17" if the far northern feature [1665-MHz F5] is included) indicates that the maser emission arises from material that is not close to the central object. The relatively small velocity spread may also refer to weak angular momentum and collapsing. This also indicates that the $\mathrm{OH}$ maser emission 


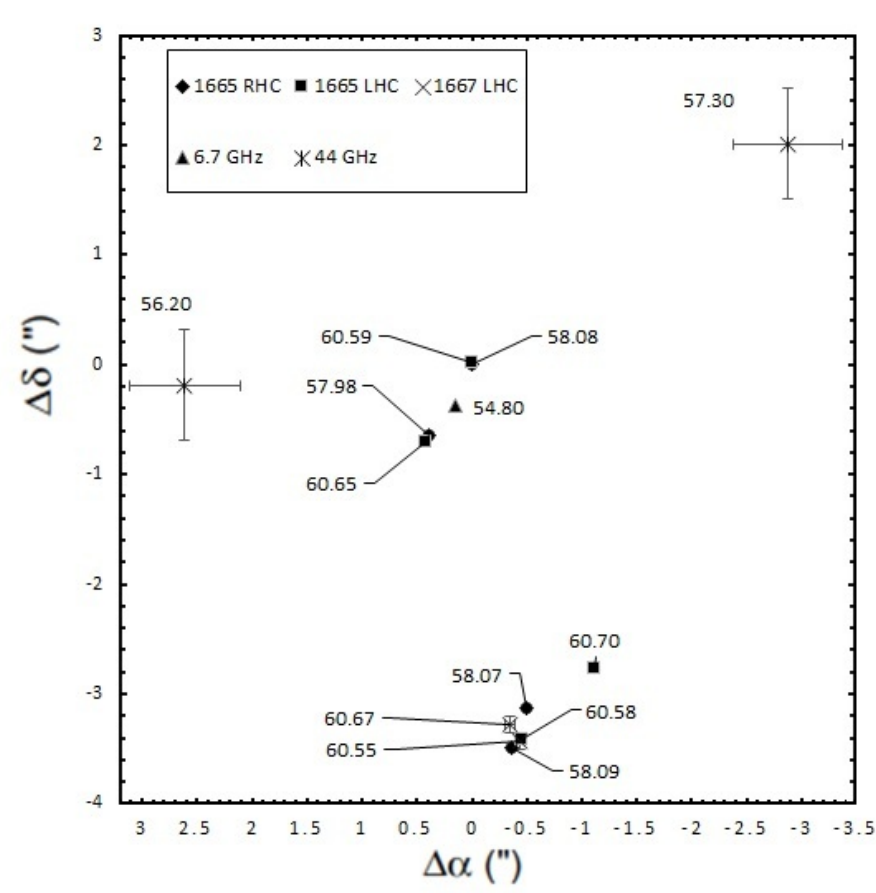

Fig. 2. Positions of the MERLIN 1.6-GHz OH maser features, VLA $44 \mathrm{GHz}$ class I methanol maser features from Kurtz et al. (2004), and MERLIN 6.7 GHz class II methanol masers (Pandian et al. 2011). The symbol that refers to each tracer is shown in the upper left corner of the plot. The plot shows close association between the $\mathrm{OH}$ masers and some 6.7-GHz Methanol maser features. The absolute positional accuracies of the MERLIN 1.6-GHz and 6.7-GHz masers are 30 mas (see Sect. 2) and 15 mas, respectively, which is smaller than the size of the symbols. The VLA absolute positional accuracies in RA and Dec associated with the $44 \mathrm{GHz}$ methanol masers are shown by the horizontal and vertical bars, respectively. The origin is at RA $(J 2000)=19^{\mathrm{h}} 11^{\mathrm{m}} 38.974^{\mathrm{s}}$ $\operatorname{Dec}(\mathrm{J} 2000)=+08^{\circ} 46^{\prime} 31.09^{\prime \prime}$. The velocities of the maser features are given. For convenience, the fifth feature of the LHC ( $\sim 14^{\prime \prime}$ offset) and a far north feature of the $44-\mathrm{GHz}\left(\sim 7^{\prime \prime}\right.$ offset) were not included in this figure.

arises from material that is not very close to the central object. This is consistent with the relatively weak excitation temperature of $10 \mathrm{~K}$ (Fontani et al. 2006) compared to the gas kinetic temperature of $40 \mathrm{~K}$ (Brand et al. 2001). Measuring the deuterium fractionation and the CO depletion factor of IRAS 19092 among ten high-mass protostellar candidates, Fontani et al. (2006) proposed two scenarios for the location of emitting gas. In the first scenario, the cold gas is distributed in an external shell that is not yet heated up by the high-mass protostellar object, which is a remnant of the parental massive starless core. In the second scenario, the cold gas is located in cold and dense cores close to the high-mass protostar but not associated with it. The observations presented here support the first scenario mentioned. This is also consistent with the association of the $\mathrm{OH}$ masers with the class I methanol masers at $44-\mathrm{GHz}$ detected by Kurtz et al. (2004). If the second scenario is true then the driving source needs still to be identified. Potential candidates are the two faint nearIR sources (2MASS $K$-Band survey) and the $1.1 \mathrm{~mm}$ sources (BGPS Survey; Fig. 2). Unfortunately, the poor positional uncertainty of these tracers does not allow us to draw any conclusion.

It is unclear whether the water and class II methanol masers at $6.7 \mathrm{MHz}$ are associated with another source or associated with the driving source. The peak velocities and velocity ranges of these other maser types are slightly different from those of 1.6- $\mathrm{GHz} \mathrm{OH}$ masers. The water maser emission peak is centred at $V=57.32 \mathrm{~km} \mathrm{~s}^{-1}$ according to Palla et al. (1991) single-dish observations, while the $6.7 \mathrm{GHz}$ methanol maser emission peak is centred at $V=55 \mathrm{~km} \mathrm{~s}^{-1}$ (Pandian et al. 2011). High angular resolution observations of the water maser emission are needed to compare its location with respect to the other maser species in the region and infer what it traces.

\subsection{Comparison with IRAS 20126}

IRAS 20126 has been mapped at same high angular resolutions by Edris et al. (2005) in the ground-state lines of $\mathrm{OH}$ masers and in the 22- $\mathrm{GHz} \mathrm{H}_{2} \mathrm{O}$ and 6.7- $\mathrm{GHz}$ (class II) $\mathrm{CH}_{3} \mathrm{OH}$ masers. Comparing the velocity range of the OH masers of IRAS 19092 with that of IRAS 20126, it is clear that towards the latter the velocity range $\left(\sim 17 \mathrm{~km} \mathrm{~s}^{-1}\right)$ is spread much more than that of the former $\left(\sim 3 \mathrm{~km} \mathrm{~s}^{-1}\right)$ although the spatial distributions of the maser features work oppositely. The $\mathrm{OH}$ maser features of IRAS 19092 trace an area of angular size three times that of IRAS 20126. The $\mathrm{OH}$ masers in IRAS 20126 trace a circumstellar disk while there is no signature of a circumstellar disk towards IRAS 19092. The strength of the magnetic field measured by a Zeeman pair in IRAS 20126 is approximately three times stronger than that of IRAS 19092. This is also consistent with the classification of Molinari et al. (1996). They classify IRAS 19092 as a source from the low sample while IRAS 20126 belongs to the high sample.

The association of $\mathrm{OH}$ masers and the two classes of methanol masers are common between these two sources. Towards IRAS 20126, the class I $44 \mathrm{GHz}$ methanol masers imaged at arcsec resolution using the VLA by Kurtz et al. (2004) are $\sim 7$ arcsec $(\simeq 0.05 \mathrm{pc})$ away from the $\mathrm{OH}$ maser position, while the class II $6.7 \mathrm{GHz}$ methanol masers are just $\simeq 0.1$ arcsec $(\simeq 0.001$ pc or $170 \mathrm{AU})$ away. Towards IRAS 19092 , the closest feature of the class I $44 \mathrm{GHz}$ methanol masers by Kurtz et al. (2004) is $\simeq 0.2 \operatorname{arcsec}(0.004 \mathrm{pc})$ away from the $\mathrm{OH}$ masers, while the class II $6.7 \mathrm{GHz}$ methanol masers are $\simeq 0.4$ arcsec $(\simeq 0.009$ pc) away.

Towards IRAS 20126, the flux density of the $\mathrm{OH}$ masers is stronger than that of IRAS 19092 . Only the $1665 \mathrm{MHz} \mathrm{OH}$ line has been detected towards IRAS 20126 while the 1665 and $1667 \mathrm{MHz}$ lines have been detected towards IRAS 19092. The presence of the two OH mainlines towards IRAS 19092 indicates lower gas temperatures and lower density according to the models of Cragg et al. (2002) and Gray et al. (1991). All this indicates that IRAS 19092 is in an earlier evolutionary stage than IRAS 20126.

\section{Conclusions}

Ground-state $\mathrm{OH}$ maser emission at 1665 and $1667 \mathrm{MHz}$ was observed towards IRAS 19092 at high angular resolution using MERLIN. Most of the $\mathrm{OH}$ maser features are spread over a region of $\sim 5^{\prime \prime}$ corresponding to $22400 \mathrm{AU}$ (or $\sim 0.1 \mathrm{pc}$ ) at a distance $4.48 \mathrm{kpc}$. We identify three Zeeman pair, indicating a magnetic field strength ranging from $\sim 4.2$ to $\sim 4.6 \mathrm{mG}$. The absence of any sign of disk, outflow, or HII region and the presence of 44-GHz class I methanol masers suggest that this source is in a very early evolutionary stage of star formation. High angular resolution observations of other tracers in the region are needed to study this SFR in more depth.

Acknowledgements. MERLIN is a national facility operated by the University of Manchester at Jodrell Bank Observatory on behalf of PPARC. 


\section{References}

Baars, J. W. M., Genzel, R., Pauliny-Toth, I. I. K., \& Witzel, A. 1977, A\&A, 61, 99

Brand, J., Cesaroni, R., Caselli, P., et al. 1994, A\&AS, 103, 541

Brand, J., Cesaroni, R., Palla, F., \& Molinari, S. 2001, A\&A 370, 230

Braz, M. A., Lepine, L. R. D., Sivagnanam, P., \& Le Squeren, A. M. 1990, A\&A 236,479

Brebner, G. C., Heaton, B., Cohen, R. J., \& Davies, S. R. 1987, MNRAS, 229, 679

Caswell, J. L. 1996, MNRAS, 279, 79

Cohen, R. J., Baart, E. E., \& Jonas, J. L. 1988, MNRAS, 231, 205

Cragg, D. M., Sobolev, A. M., \& Godfrey, P. D. 2002, MNRAS, 331, 521

Diamond, P. J., Garrington, S. T., Gunn, A. G., et al. 2003, MERLIN User Guide, ver. 3

Edris, K. A., Fuller, G. A., Cohen, R. J., \& Etoka, S. 2005, A\&A, 343, 213

Edris, K. A., Fuller, G. A., \& Cohen, R. J. 2007, A\&A, 465, 865 (EFC07)

Ellingsen, S. P. 2006, ApJ, 638, 241

Elitzur, M. 1996, ApJ, 457, 415

Etoka, S., Gray, M. D., \& Fuller, G. A. 2012, MNRAS, 423, 647

Faustini, F., Molinari, S., Testi, L., \& Brand, J. 2009, A\&A, 503, 801

Fontani, F., Caselli, P., Crapsi, A., et al. 2006, A\&A, 460, 709

Fontani, F., Cesaroni, R., \& Furuya, R. S. 2010, A\&A, 517, A56
Fuller, G. A., Zijlstra, A. A., \& Williams, S. J. 2001, ApJ, 555, L125 Garay, G., \& Lizano, S. 1999, PASP, 111, 1049

Garcia-Barreto, J. A., Burke, B. F., Reid, M. J., et al. 1988, ApJ, 326, 954 Gasiprong, N., Cohen, R. J., \& Hutawarakorn, B. 2002, MNRAS, 336, 47 Gaume, R. A., \& Mutel, R. L. 1987, ApJS, 65, 193

Gray, M. D., Doel, R. C., \& Field, D. 1991, MNRAS, 252, 30 Gray, M. D., Hutawarakorn, B., \& Cohen, R. J. 2003, MNRAS, 343, 1067 Hutawarakorn, B., \& Cohen, R. J. 1999, MNRAS, 303, 845

Hutawarakorn, B., Cohen, R. J., \& Brebner, G. C. 2002, MNRAS, 330, 349 Kurtz, S., Hofner, P., \& Alvarez, C. V. 2004, ApJS, 155, 149

MacLeod, G., van der Walt, D. J., North, A., et al. 1998, AJ, 116, 2936 Molinari, S., Brand, J., Cesaroni, R., \& Palla, F. 1996, A\&A, 308, 573 Molinari, S., Brand, J., Cesaroni, R., et al. 1998, A\&A, 336, 339

Molinari, S., Brand, J., Cesaroni, R., \& Palla, F. 2000, A\&A, 355, 617 Palla, F., Brand, J., Cesaroni, R., et al. 1991, A\&A, 246, 249

Pandian, J. D., Momjian, E., Xu, Y., Menten, K. M., \& Goldsmith, P. F. 2011, ApJ, 730, 55

Rosolowsky, E., Dunham, M. K., Ginsburg, A., et al. 2010, ApJS, 188, 123

Szymczak, M., Hrynek, G., \& Kus, A. J. 2000, A\&AS, 143, 269

Varricatt, W. P., Davis, C. J., Ramsay, S., \& Todd, S. P. 2010, MNRAS, 404, 661 Wood, D. O. S., \& Churchwell, E. 1989, ApJS, 83, 119

Wu,Y., Henkel, C., Xue, R., Guan, X., \& Miller, M. 2007, ApJ, 669, 37

Zhang, Q., Hunter, T. R., Brand, J., et al. 2005, ApJ, 625, 864 